\title{
Bargaining Based Pairwise Cooperative Spectrum Sensing for Cognitive Radio Networks
}

\author{
Miao Pan $\dagger \ddagger$ \\ ${ }^{\dagger}$ Department of Electrical and Computer Engineering \\ University of Florida, Gainesville, FL 32611 USA \\ miaopan@ufl.edu
}

\author{
Yuguang Fang $\ddagger$ \\ ${ }^{\ddagger}$ Department of Electrical and Computer Engineering \\ University of Florida, Gainesville, FL 32611 USA \\ fang@ece.ufl.edu
}

\begin{abstract}
Cognitive Radio (CR) network is promising for military usage since it enables the CR users in the field to sense and take the opportunistic use of vacant frequency bands if no primary users are present. As a key component of the CR technology, spectrum sensing has been investigated actively in existing literature. Among all the problems related to spectrum sensing, two of them seem to be challenging: first, CR users are likely to miss the detection of the primary users, resulting in low sensing accuracy; second, to guarantee the frequency bands are available, CR users have to spend a large amount of time sensing those bands, which inevitably decreases their data transmission opportunities, leading to the so-called sensing efficiency problem.

This paper proposes a bargaining based pairwise cooperative spectrum sensing (BBPCSS) scheme as a possible solution. In order to increase the sensing accuracy, the CR users are grouped into pairs for cooperative sensing instead of individual sensing. In each pair, rational $C R$ users can bargain with each other over the sensing time division, and thus save the sensing time for data transmission. Our analysis and simulation results suggest that BBPCSS effectively improves the detection probability and enhances the sensing efficiency.
\end{abstract}

Index Terms-cognitive radio, spectrum sensing, pairwise cooperation, bargain

\section{INTRODUCTION}

Recent years have witnessed the breakneck proliferation of wireless devices and rapid growth of wireless services. In parallel with that, the ever-increasing demand for radio spectrum continues to stretch this inherently limited natural resource. Within the current regulatory framework, most of the spectrum is allocated to licensed users for exclusive use by government agencies (e.g. Federal Communication Commission). It is becoming exceedingly difficult to find available bands to either deploy new services or to enhance the existing ones. On the other hand, measurement has indicated that over $60 \%$ of the licensed spectrum below $6 \mathrm{GHz}$ is under utilization [1]-[3]. To take advantage of the so-called "white space" in existing bands [4], Cognitive Radio (CR) is proposed as a promising technology to implement the dynamic spectrum access and improve the spectral efficiency. Since CR users have the capability to sense, access to, and transmit on the licensed frequency bands opportunistically when the primary user is inactive [5], [6], the potential usage of CR network has

This work was supported by the National Science Foundation under grants CNS-0721744 and DBI-0529012. been recognized in the commercial sector [7] as well as in the public safety and military communications [8].

Since CR users cannot interfere with the operation of the primary user, they must detect the presence of the primary user in a very short time and must vacate the band as long as the primary user returns. So one of the challenges that confronts this technology is how do the CR users carry out accurate spectrum sensing. Among the several known PHYlayer sensing methods, feature detection, matched-filter detection, and energy detection are three prominent schemes for sensing the primary user [9]-[11]. Feature detection exploits the inherent periodicity in the received signal to detect the primary signals with a particular modulation type [12]. As illustrated in [13], the matched-filter detection is the optimal sensing choice in stationary Gaussian noise, when the structure of primary signal is known to the CR users. Despite the difficulty in circuitry design and implementation, there may be many cases in practice where feature detection and matchedfilter detection are ruled out due to the lack of knowledge about the primary signal's information. Thus energy detection [14], [15] is preferred as a common and feasible method for detection of unknown signals in noise. However, the performance of energy detection is inclined to degrade in shadowing/fading environments.

In order to mitigate the multi-path fading and shadowing effects of the individual sensing, cooperation among CR users has been investigated in [9], [16], [17]. The better performance of cooperative sensing can be achieved either by synchronizing different CR users to sense together [13], or by scheduling different CR users to sense sequentially over different time slots [12]. Correspondingly, a centralized scheduler is needed to coordinate CR users. But the number of the CR users involved in the cooperation may be large and time-varying, which incurs great computational complexity and makes the centralized based algorithms difficult to be implemented.

In [18], [19], a distributed cooperative spectrum sensing scheme for the CR network is provided. According to their locations away from the primary user, CR users are grouped into pairs. In the cooperative sensing pairs, the CR user near the primary user senses the band and relays the detected information to the one faraway from the primary user. Although this method increases the sensing accuracy, and makes the cooperation among $\mathrm{CR}$ users more practical and scalable, the 
hardware limitations of CR users introduce another critical issue on spectrum sensing. To preclude interference to the primary user, in ideal cases, CR users should monitor the spectrum continuously through the radio frequency (RF) frontend. But in reality, current RF front-ends cannot perform sensing and transmission at the same time. So there is tradeoff between sensing accuracy (i.e. interference avoiding) and sensing efficiency (i.e. CR users have more opportunities of transmission) [12], [6]. Especially for cooperative sensing, it is important for CR users to choose their strategies for cooperation, i.e. how to properly divide their time between sensing and transmission, which have a crucial impact on the performance of the CR network.

In this paper, we are dedicated to designing a reasonable cooperative sensing scheme to give a comprehensive attention to both interference avoidance and sensing efficiency improvement. A bargaining based cooperative spectrum sensing (BBPCSS) scheme for CR networks is proposed and four contributions are made. Firstly, similar to [18], a spectrum sensing framework of pairwise cooperation is developed. The $\mathrm{CR}$ users are endowed with high intelligence [17] and rational CR users in each pair can negotiate with each other over the sensing time division. In this way, the CR user is able to take advantage of his partner's sensing time for his own data transmission. Secondly, taking both sensing accuracy and sensing efficiency into account, we put forward a novel utility function for the evaluation. Thirdly, inspired by bargaining game in microeconomics [20], we apply the Rubinstein-Stahl's theory into strategy design of the CR users in the pairwise cooperation to maximize their utilities. Finally, we do simulations in different environments, and the results reveal that BBPCSS can improve the detection probability and enhance the sensing efficiency significantly.

Remainder of the paper is organized as follows.In section II, system model is outlined and related concepts are introduced. The Rubinstein-Stahl bargaining theory and its application in BBPCSS are illustrated in section III. In section IV, CR users' strategies and expected utility are developed. The simulations and analysis are demonstrated in section V. Finally, some concluding remarks are made in section VI.

\section{SYSTEM MODEL}

In this section, the framework of the pairwise cooperative sensing is described, related models [6], [12], [14], [15], [18] for spectrum sensing in CR networks are introduced, and the utility function of CR users is defined. All these models and definitions will be used throughout the paper.

\section{A. System Description}

Consider a network with two CR users, ${ }^{1} C_{1}$ and $C_{2}$, who send data to different base stations at the same transmission rate $R$. If the primary user starts using the spectrum band, CR users need to vacate the band as soon as possible to avoid interference to the primary user. As to the CR user far away

\footnotetext{
${ }^{1}$ The two user CR network can easily be extended to a multiuser CR network [18], [19]
}

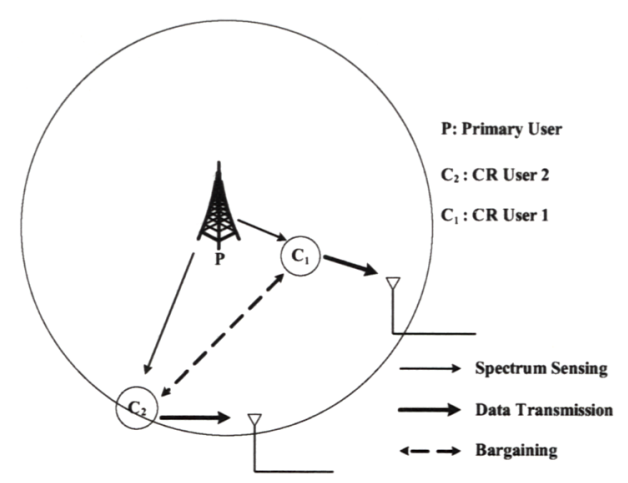

(a) Pairwise cooperation in CR networks

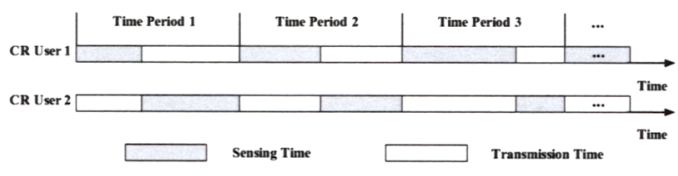

(b) Time division between sensing and data transmission

Fig. 1. The framework of pairwise cooperative spectrum sensing

from the primary user, say $C_{2}$, the signal received from the primary user is so weak that $C_{2}$ has trouble in detecting the presence of the primary user in a short time. Compared with $C_{2}, C_{1}$ has much better sensing accuracy since he is closer to the primary user as shown in Fig. 1(a). So, intuitively, if $C_{1}$ can always monitor the spectrum band and relay all the detected information to $C_{2}$, the sensing problem of $C_{2}$ is settled. However, $C_{1}$ is not able to continuously sense the spectrum band since he also needs time to transmit data, which makes the gratuitous manner of cooperation infeasible.

In this paper, we allow the CR users to cooperate according to their bargaining results. To facilitate the process, the time is divided into periods. In each time period $T$, the intelligent CR users, $C_{1}$ and $C_{2}$, need to negotiate with each other on the sensing time division through common control channel (CCC) [22]. Once the agreement is reached, the CR users sense the spectrum band and transmit data in turns according to the agreement, as depicted in Fig. 1(b).

\section{B. Related Models for Sensing}

1) Energy Detection Model: To tell the primary signal from the other signals as well as noise, the energy of the different received signals should be measured. Typically, in energy detection [15], received signals are filtered to a selected bandwidth $W$, passed through a square law detector, and integrated for a time period $T_{s}$. Then, the output of the integrator $Y$ is compared with a threshold $\lambda$ to decide whether the primary user is present or not. The detection probability $P_{d}$ and false alarm probability $P_{f}$ in MAP-based energy detection can be expressed as [13]-[15],

$$
\begin{gathered}
P_{d}\left(W, T_{s}\right)=P\left\{Y>\lambda \mid H_{1}\right\}=Q\left(\frac{\lambda-2 T_{s} W\left(P_{n}+P_{s}\right)}{\sqrt{4 T_{s} W\left(P_{n}+P_{s}\right)^{2}}}\right) \\
P_{f}\left(W, T_{s}\right)=P\left\{Y>\lambda \mid H_{0}\right\}=Q\left(\frac{\lambda-2 T_{s} W P_{n}}{\sqrt{4 T_{s} W P_{n}^{2}}}\right)
\end{gathered}
$$


where $H_{0}$ represents the hypothesis of "no signal transmitted", and $H_{1}$ represents "signal transmitted". $P_{s}$ and $P_{n}$ are the received signal power and received noise power, respectively.

Obviously, CR users have different detection and false alarm probabilities depending on the parameters $W, T_{s}$ and $\lambda$. Note that the decision threshold $\lambda$ is inherently determined by $W$ and $T_{s}$ for the MAP-based energy detection, as explained in [12], [15]. Given the bandwidth of the detector $W$ is fixed, the $\mathrm{Q}$ function monotonously increases with $T_{s}$, i.e. detection probability can be improved by increasing the integration time $T_{s}$ [12], [15].

2) Experienced Model of Primary User Activities: Since primary user activities are closely related to the performance of CR networks, the estimation of this activity is essential for spectrum sensing. In this paper, we assume that primary user activities can be modeled as exponentially distributed interarrivals [21].

In this experienced model, the primary user traffic can be regarded as a two state birth-death process with death rate $\alpha$ and birth rate $\beta$. An ON (Busy) state represents the period used by primary users and an OFF (Idle) state represents the unused period. The probability with primary user "ON" can be obtained as $\frac{\alpha}{(\alpha+\beta)}$. Correspondingly, the probability with primary user "OFF" is $\frac{\beta}{(\alpha+\beta)}[21]$.

\section{Utility Function}

With the assumption that the CR users are rational, either of them would like to reserve as much time as possible for his own data transmission, and make the cooperation pair have as high probability as possible to detect the presence of the primary user. Therefore, both CR users have a joint consideration of sensing efficiency and sensing accuracy. In order to reflect the double targets of the CR users, the utility function should be defined as,

$$
U_{i}\left(x_{i}\right)=R T\left(1-x_{i}\right)\left(\sum_{k \in N} T x_{k} P_{d}\left(T x_{k}\right)\right) \forall i \in N
$$

where $N=\{1,2\}$ and $x_{i}=\frac{T_{s}^{i}}{T}$ represents $C_{i}$ 's share of sensing time.

\section{COOPERATIVE SENSING VIA BARGAINING}

Based on the assumptions and models above, we fit Rubinstein-Stahl bargaining game into the time division of pairwise cooperative spectrum sensing in this section.

\section{A. Rubinstein-Stahl Bargaining in BBPCSS}

Rubinstein-Stahl bargaining was proposed as a solution to the problem that two players (player $i(i \in\{1,2\})$ ) are bargaining over the division of a "cake" of size 1 [20]. They negotiate with each other by proposing offers alternately. An agreement is a pair $\left(x_{1}, x_{2}\right)$, in which $x_{i}$ is player $i$ 's share of the cake. The set of possible agreements is,

$$
X=\left\{\left(x_{1}, x_{2} \in \Re^{2}: x_{1}+x_{2} \leq 1, x_{i} \geq 0, \forall i \in\{1,2\}\right)\right\}(4)
$$

Similar to Rubinstein-Stahl's game, the two CR users in pairwise cooperative sensing can be regarded as two players bargaining over their sensing time proportion of the time period to maximize their utilities. We interpret $x_{i}$ as the amount of time that CR user $i$ obtains. That is, CR user $i$ prefers $x \in X$ to $y \in X$ iff $U_{i}\left(x_{i}\right)>U_{i}\left(y_{i}\right)$

\section{B. Bargaining Procedure}

The sensing time bargaining procedure between pairwise cooperating CR users is as follows. In round $0, C_{1}$ begins by making a offer, say $(x, 1-x)$, where $0 \leq x \leq 1$ represents the part of the time period that he demands for himself. $C_{2}$ can then either accept or reject this offer. If he accepts, the agreement is reached; if he rejects, a period of bargaining time $\triangle>0$ must elapse. When they come back to the negotiation table at round 1 , the roles are reversed so that $C_{2}$ will make a new offer $(y, 1-y)$, where $0 \leq y \leq 1$ is the fraction of the time unit that he offers to $C_{1} . C_{1}$ must then either accept the new offer, in which case bargaining ends with $(y, 1-y)$ as the agreement; or reject it, in which case time $\triangle$ must elapse before $C_{1}$ makes a new proposal. In round 2(i.e. at time $2 \triangle), C_{1}$ proposes $(z, 1-z)$, to which $C_{2}$ must respond, and so on. The bargaining may be finite horizon game or infinite horizon game. The $M$-round finite horizon game imposes the disagreement outcome, with zero payoffs, after $M$ proposals have been rejected. On the other hand, in the infinite horizon version, there is always a new offer in the next round after an offer is rejected.

Note that bargaining process is time-costing. The longer bargaining lasts, the less utility the CR users achieve. That is to say, both of CR users discount the future at a rate. Let $\delta_{i} \in[0,1)$ be the per round discount factor for $C_{i}$. If the CR users reach an agreement at round $\mathrm{t}$ (i.e. at time $t \triangle$ ) on a division that gives $C_{i}$ a share $\delta_{i}^{t} x_{i}$ of the time period, then $C_{i}$ 's payoff is $U\left(\delta_{i}^{t} x_{i}\right)$. If CR users never reach an agreement, then CR user's utility is zero. So, the utility of $C_{i}$ is increasing with the share $x_{i}$, and decreasing with delay $t \triangle$, with which the agreement happens. In addition, the utility is subject to the time discount factor, which is also called bargaining patience of the CR users. A more patient CR user (i.e. the CR user with larger $\delta$ ) benefits more from the negotiations. Thus, the bargaining between cooperative sensing CR users can also be considered as a game depending on the patience of the individual $\mathrm{CR}$ user.

\section{Bargaining Patience}

As we have seen, the patience plays an important role in the alternating bargaining process. The better patience the $C R$ user has, the better utility he attains. Traditionally, the bargaining patience factor in the Rubinstein-Stahl's game can be defined as follows,

$$
\delta_{i}=e^{-r_{i} \triangle}
$$

where $r_{i}>0$ is player $i$ 's bargaining patience rate. Given the $\triangle$ is fixed, $\delta_{i}$ is monotonic decreasing with $r_{i}$.

In the scenario of pairwise cooperative sensing, different $C R$ users have different discount rates. Intuitively, the discount rate of the CR user is not supposed to a constant but a variable 


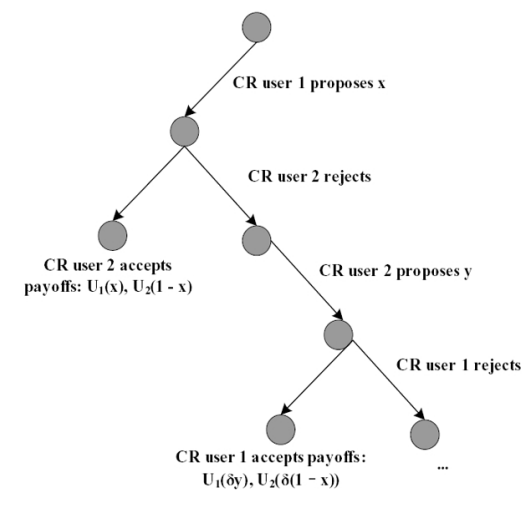

Fig. 2. Bargaining procedure in BBPCSS

changing over the distance $d$ between the CR user and the primary user in the CR network. For example, suppose there is CR user $C_{i}$ moving within the detectable boundary of the primary user. If he is moving away from the location of the primary user, he will gradually lose his patience. The reason is that $C_{i}$ is increasingly eager to get help from the other CR user in the cooperation sensing pair since his received power of the primary user is attenuating due to the path loss. Therefore, his bargaining patience rate $r_{i}$ will increase. On the contrary, if he is moving toward the location of the primary user, he will have more and more patience and his bargaining patience rate $r_{i}$ will decrease. So, $r_{i} \propto d_{i}$. To be simple, $r_{i}$ can be expressed as $r_{i}=k d_{i}$, where $k$ is a constant [13], [26].

\section{BARGAINING STRATEGIES AND EXPECTED UTILITY}

In order to best their utilities in the agreement, the CR users need to devise their strategies in the bargaining. In this section, we introduce the subgame perfect equilibrium (SPE) in extensive-form game, and elaborate on the optimal bargaining strategy with complete information for the CR users in BBPCSS. In addition, we formulate the expected utilities of the CR users with regards to the activities of the primary user.

\section{A. Bargaining Strategies in BBPCSS}

Let $S$ denote the strategy set of the CR users. To develop the optimal bargaining strategies $s^{*}=\left(s_{1}^{*}, s_{2}^{*}\right) \in S$ for the CR users, two assumptions are needed: (1) complete knowledge of the circumstances in which BBPCSS is carried out and (2) full rationality of the $\mathrm{CR}$ users.

The first assumption implies that the rules of the bargaining game and the preferences (i.e., the utility functions) and beliefs (i.e., the patience factor $\delta$ ) of the $\mathrm{CR}$ users are common knowledge. As mentioned in in Sec. III- C, $\delta$ is determined by the distance between the CR user and the primary user, which can be easily calculated from the location of that CR user. Just like in [18], [19], the location information as well as the utility function of the individual CR user can be shared in a distributed manner. The second assumption relates to the need for common knowledge on how CR users reason. It is assumed that CR users maximize their utilities given their beliefs, and have enough computational capacity and memory.

Using these assumptions, the CR users seek to find the strategy to set an equilibrium in the bargaining, which can maximize their own utilities. Recalling the bargaining process, the CR users can make decisions sequentially and finish the negotiation with an agreement at various stages of the bargaining. These features characterize the bargaining in BBPCSS as an extensive-form game with a tree structure as illustrated in Fig. 2. The equilibria in such a game are defined as the follows:

Definition. Strategies in an extensive-form game are in Subgame Perfect Equilibrium (SPE) if the strategies constitute a Nash equilibrium at every decision point.

Besides, SPE in extensive-form game has the following two properties,

- (No Delay) All equilibrium offers are accepted.

- (Stationarity) A CR user always makes the same offer in equilibrium.

Let $x_{1}^{*}$ denote the equilibrium offer by $C_{1}$ in BBPCSS. Given properties above, there exists a value $x_{1}^{*}$ such that, in the SPE of this game, $C_{1}$ proposes $x_{1}^{*}$ at the first stage and rational $C_{2}$ accepts. In fact, the current present value of rejecting an offer $x_{1}^{*}$, is $\delta_{2} x_{2}^{*}$ for $C_{2}$. This implies that in equilibrium

$$
U_{2}\left(1-x_{1}^{*}\right)=U_{2}\left(\delta_{2}\left(x_{2}^{*}\right)\right)
$$

Similarly, let $x_{2}^{*}$ denote the equilibrium offer by $C_{2}$ in BBPCSS. If $C_{2}$ proposes $x_{2}^{*}$ at the first stage, there is,

$$
U_{1}\left(1-x_{2}^{*}\right)=U_{1}\left(\delta_{1}\left(x_{1}^{*}\right)\right)
$$

Therefore, there is a unique solution

$$
\begin{aligned}
& x_{1}^{*}=\left(1-\delta_{2}\right) /\left(1-\delta_{1} \delta_{2}\right) \\
& x_{2}^{*}=\left(1-\delta_{1}\right) /\left(1-\delta_{1} \delta_{2}\right)
\end{aligned}
$$

Thus, at least one SPE satisfies the two properties. But we still have to verify uniqueness of such an equilibrium. Let $\Gamma_{i}$ denote any subgame that starts with $C_{i}$ making an offer. In Rubinstein's work [20], the following lemma is proved, which guarantees the SPE to be unique in BBPCSS.

Lemma. There exists a unique SPE payoff profile of $\Gamma_{i}$ given by $\left(x_{i}^{*}, 1-x_{i}^{*}\right)$

So, the corresponding strategy profile $s^{*}=\left(s_{1}^{*}, s_{2}^{*}\right)$ can be obtained as the following:

$C_{1}$ : Always offer $x_{1}^{*}$, accept any $x_{2}$ with $\left(1-x_{2}\right) \geq \delta_{1} x_{1}^{*}$.

$C_{2}$ : Always offer $x_{2}^{*}$, accept any $x_{1}$ with $\left(1-x_{1}\right) \geq \delta_{2} x_{2}^{*}$.

If both CR users use their SPE strategies, agreement will be achieved in the first turn itself. Without the time consumption on alternating the offers, $s_{i}^{*}$ (i.e., offering $x_{i}^{*}$ ) should be deemed as the optimal bargaining strategy for $C_{i}$.

Although the unique SPE payoff exists, bargaining starting with different $\mathrm{CR}$ users will lead to different sensing time partition of the time period. For instance, if $C_{1}$ initiates 
the bargaining with the optimal strategy, the agreement is $\left(\frac{1-\delta_{2}}{1-\delta_{1} \delta_{2}}, \frac{\delta_{2}\left(1-\delta_{1}\right)}{1-\delta_{1} \delta_{2}}\right)$. However, if $C_{2}$ initiates the bargaining with the optimal strategy, the agreement is $\left(\frac{\delta_{1}\left(1-\delta_{2}\right)}{1-\delta_{1} \delta_{2}}, \frac{1-\delta_{1}}{1-\delta_{1} \delta_{2}}\right)$. We compare the two SPE results of bargaining initialized by different $\mathrm{CR}$ users. For $0 \leq \delta_{i} \leq 1$, it is obvious that $\frac{1-\delta_{2}}{1-\delta_{1} \delta_{2}} \geq \frac{\delta_{1}\left(1-\delta_{2}\right)}{1-\delta_{1} \delta_{2}}$ for $C_{1}$ and $\left.\frac{\overline{\delta_{2}}\left(1-\delta_{1}\right)}{1-\delta_{1} \delta_{2}}\right) \leq \frac{1-\delta_{1}}{1-\delta_{1} \delta_{2}}$ for $C_{2}$. To put it in another way, there is a first mover advantage for CR users in BBPCSS.

\section{B. Expected Utility}

Due to the existence of the first mover advantage in the SPE of alternating offers bargaining, CR users in BBPCSS choose to apply different equilibrium strategies according to the prediction of the primary user's activities.

If the primary user is predicted to be "ON" during this period of time, the CR user closer to the primary user, who has larger detection probability, will take the first mover advantage to reach the SPE. Otherwise, if the primary user is predicted to be "OFF" during the period of time, the CR user farther away from the primary user, who has smaller detection probability, will take the first mover advantage to reach the SPE.

Without loss of generality, we assume $C_{1}$ is closer to the primary user. With the model of primary user activities from Sec. II- C, we can obtain the expected utility of the CR user in arbitrary time period as,

$$
\begin{aligned}
& U_{E X 1}^{*}=U_{1}\left(x_{1}^{*}\right) P_{o n}+U_{1}\left(1-x_{2}^{*}\right) P_{o f f} \\
& U_{E X 2}^{*}=U_{2}\left(1-x_{1}^{*}\right) P_{o n}+U_{2}\left(x_{2}^{*}\right) P_{o f f}
\end{aligned}
$$

\section{SIMULATION AND ANALYSIS}

\section{A. Simulation Environments}

We carry out simulations to test and demonstrate the performance of our BBPCSS scheme. In the simulations, a pair of CR users, who have the same transmission ability and detection features, are located in a circle area with the radius of $200 \mathrm{~m}$. The primary user [22] is located at the center of the CR network as shown in Fig. 1. Since the detection probabilities change over the sensing terminal's locations, they should be recalculated when the CR user moves to a different place. To utilize the physical locations of the CR users, we calculate these detection probabilities based on the log-normal shadowing path loss model [23], [24]. The lognormal shadowing path loss model can be given as,

$$
P L(d)=\overline{P L}(d)+X_{\sigma}=\overline{P L}\left(d_{0}\right)+10 l \log \left(\frac{d}{d_{0}}\right)+X_{\sigma}
$$

where $d$ is the transmitter-receiver distance, $P L(d)$ is the path loss as a function of $d, \overline{P L}(d)$ is the mean of $P L(d)$, $X_{\sigma}$ is a zero-mean Gaussian distributed random variable with standard deviation $\sigma, d_{0}$ is a close-in reference distance which is typically $1 m$ away from the transmitter [24], and $l$ is the path loss exponent which indicates the rate at which the path loss increases with distance. All items in the equation are in dB.
TABLE I

Simulation PARAMETERS

\begin{tabular}{|l|l|}
\hline \hline Time Period: $T(\mathrm{~s})$ & 10 \\
\hline Bandwidth: $W(\mathrm{KHz})$ & 100 \\
\hline Transmission Rate: $R$ (Mbps) & 0.1 \\
\hline Death Rate: $\alpha$ & 0.3 \\
\hline Birth Rate: $\beta$ & 0.4 \\
\hline Correlation Constant: $k$ & 0.002 \\
\hline \hline
\end{tabular}

Thus, the received power $P_{s}$ in equation (1) can be denoted as,

$$
P_{s}=P_{t}-P L(d)=P_{t}-\overline{P L}(d)-X_{\sigma}
$$

where $P_{t}$ is the transmitted power of the primary user, and both $P_{s}$ and $P_{t}$ are in $\mathrm{dB}$.

Here, the $\overline{P L}(d)$ employs the HATA model [24], which has been suggested by the 802.22 working group as the path loss model for a representative CR network environment [25]. This model has several versions for different environments, such as rural areas, urban areas, and open areas. We adopt the one for the rural environments since the CR network is most likely to be applied in rural areas [22]. The model can be expressed as,

$$
\begin{aligned}
\overline{P L}(d)= & 27.77+9.39 \log f_{c}-4.78\left(\log f_{c}\right)^{2} \\
& -13.82 \log h_{t e}-\left(1.1 \log f_{c}-0.7\right) h_{r e} \\
& +\left(44.9-6.55 \log h_{t e}\right) \log d
\end{aligned}
$$

where $f_{c}$ is the primary signal frequency, $h_{t e}$ is the effective antenna height of the primary user in meters, and $h_{r e}$ is the effective antenna height of the CR users in meters, and $d$ is the distance between the primary user and CR users in kilometers. All the other items in the equation above are in $\mathrm{dB}$. In our simulations, we assume that the primary user works at the UHF frequency of $423 \mathrm{MHz}$, and the antenna heights of the primary user and the CR users are $100 \mathrm{~m}$ and $1 \mathrm{~m}$, respectively. The effective isotropic radiated power (EIRP) of the primary user is assumed to be $50 \mathrm{dBw}$. For the CR users, a simple energy detector is adopted. Each receiver has a typical sensitivity of $-94 \mathrm{dbm}$, which is the minimum power for a signal to be detected.

For illustrative purposes, we simplify the movement of the two CR users within the range of the network area and make the following assumptions: $C_{1}$ is fixed at the location $100 \mathrm{~m}$ away from the primary user and he does not move; $C_{2}$, in the same semicircle with $C_{1}$, is $50 \mathrm{~m}$ away from the primary user and moves at the uniform speed of $5 \mathrm{~m} / \mathrm{s}$ away from the primary user. Besides, note that in all the simulations, the $P_{f}$ is held constant at $0.1 \%$ while the transmission time, sensing time, and $S N R$ in the equations are varied. Some other related parameters for the simulations are listed in Table I.

\section{B. Results and Analysis}

As an important criterion for evaluating the performance of the BBPCSS, the utilities of both CR users are extensively investigated in the simulations. 


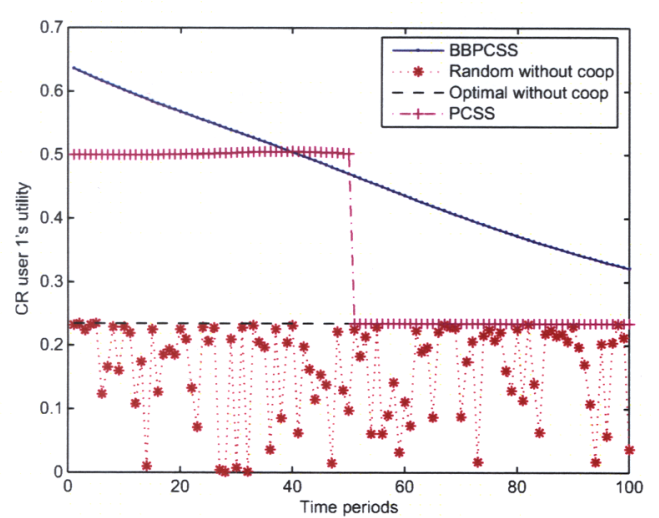

(a) $C_{1}$ 's performance with different schemes

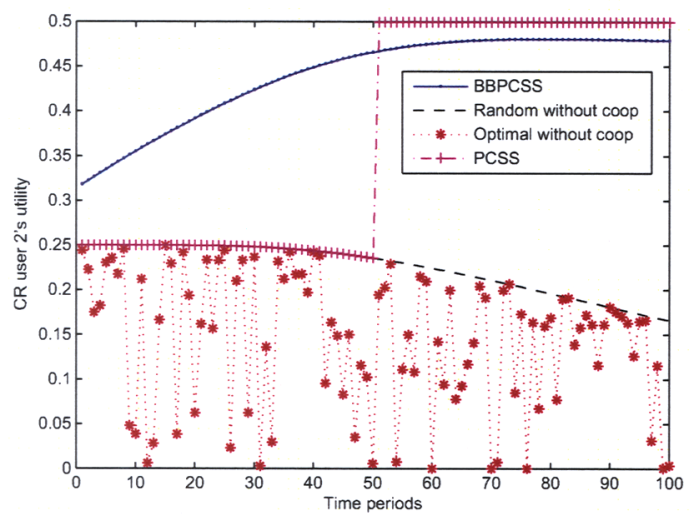

(b) $C_{2}$ 's performance with different schemes

Fig. 3. The utility of CR users with four different schemes

Fig. 3 (a) and (b) depict the comparisons of the CR users' utilities with four different sensing schemes, i.e. optimal spectrum sensing without cooperation ${ }^{2}$, random spectrum sensing without cooperation ${ }^{3}$, pairwise cooperative spectrum sensing (PCSS) [18], and our proposed BBPCSS. It is obvious that the CR users' utilities of the cooperation sensing schemes are far better than those of the individual sensing schemes. As to PCSS, $C_{2}$ benefits a lot from the cooperation when $C_{1}$ is closer to the primary user, and vice versa. The reason is that in PCSS, the CR user near to the primary user has to take all the responsibility to detect the primary user and relay the information to the other one. Assuming the CR user closer to the primary user is rational, he may refuse to provide the relay offer. Different from PCSS, the proposed BBPCSS allow for the bargaining of sensing time sharing between the two CR users according to their locations as mentioned above. That is to say, the CR users monitor the spectrum in turns with BBPCSS, and there is only one user doing the sensing in PCSS. Consequently, our BBPCSS outperforms PCSS during

\footnotetext{
${ }^{2}$ Ideally, the individual CR user optimally balances time between sensing and transmission to obtain the best utility.

${ }^{3}$ The individual CR user randomly allocates time between sensing and transmission.
}

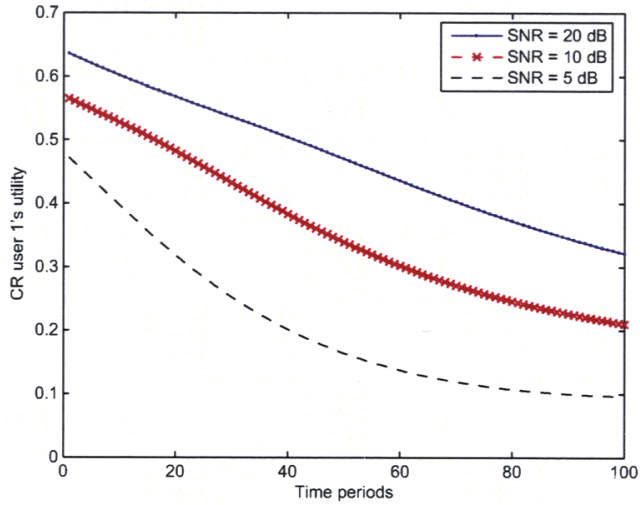

(a) $C_{1}$ 's performance with different SNRs

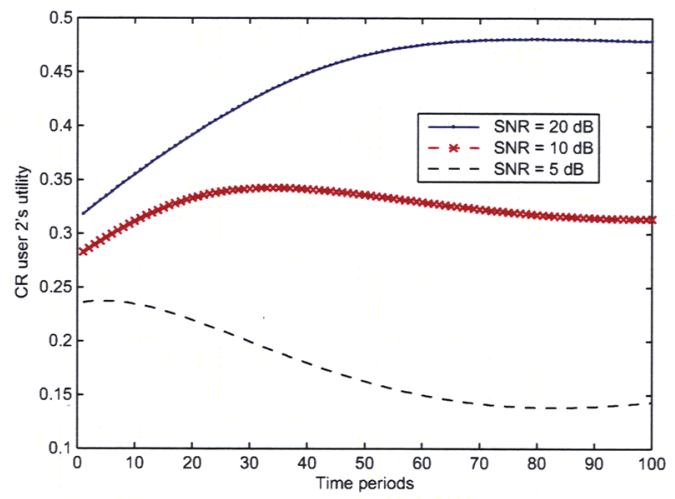

(b) $C_{2}$ 's performance with different SNRs

Fig. 4. The utility of CR users with three different SNRs

most simulation periods. Moreover, in BBPCSS, better SNR leads to more utilities of both CR users as illustrated in Fig. 4.

The more utilities are earned, the more data are transmitted, and the better detection probabilities are achieved. It can be logically deduced that the CR users trunking increase of utility should be accompanied by the improving of sensing accuracy, sensing efficiency, and spectrum utilization.

\section{CONCLUSION AND FUTURE WORK}

Spectrum sensing is the key enabling technology for the applications of CR networks in commercial, public safety, and particularly military areas. Interference avoidance and the sensing efficiency improvement are two important challenges for spectrum sensing. In this paper, we presented a reasonable cooperative sensing scheme using bargaining theory to give a joint consideration of both issues. We brought forward the framework of pairwise cooperation spectrum sensing by allowing for negotiations between CR users over the sensing time division. Considering both detection probability and sensing efficiency, we then introduced a utility function for evaluation. Furthermore, we applied the Rubinstein-Stahl's bargaining theory into strategy design of the CR users to maximize their 
utility in the pairwise cooperation sensing. Also, we compared our scheme with previous schemes and did simulations in different environments. The simulation results show that the proposed BBPCSS indeed improves the sensing accuracy and enhances the sensing efficiency.

In this paper, all the CR users are assumed to be identical and their movement is simplified. Moreover, only distance between the CR user and the primary one is regarded as the factor affecting the bargaining results. In the future, we wish to develop cooperative protocols where the mobility of the CR users is not limited, and more features of the CR users, such as different received signal powers and various data transmission rates, are involved in.

\section{REFERENCES}

[1] Federal Communications Commision, "Spectrum policy task force report," Report, Et docket No. 02-135, Nov. 2002.

[2] J. Yang, Spatial channel characterization for cognitive radios, MS Thesis, UC Berkeley, 2004.

[3] I. F. Akyildiz, W. Lee, M. Vuran, and M. Shantidev, "NeXt generation/ dynamic spectrum access/ cognitive radio wireless networks: a survey", Computer Networks Journal (Elsevier), vol. 50, pp.2127-2159, Sep. 2006.

[4] M. Marcus, "Real time spectrum markets and interruptible spectrum: new concepts of spectrum use enabled by cognitive radio," in Proc. of First IEEE International Symposium on Dynamic Spectrum Access Networks, DySPAN '05, pp. 512-517, Nov. 2005.

[5] X. Liu and S. Shankar, "Sensing-based opportunistic channel access", ACM Journal on Mobile Networks and Applications (MONET), vol. 11, pp. 577-591, Feb. 2006.

[6] H. Kim and K. Shin, "Efficient Discovery of Spectrum Opportunities with MAC-Layer Sensing in Cognitive Radio Networks", IEEE Transactions on Mobile Computing, vol. 7, No. 5, pp. 533-545, May 2008

[7] Joint Tactical Radio System, http://enterprise.spawar.navy.mil/.

[8] SAFECOM 1 http://www.safecomprogram.gov/SAFECOM/.

[9] D. Cabric, S.M. Mishra, and R.W. Brodersen, "Implementation Issues in Spectrum Sensing for Cognitive Radios," in Proc. of Asilomar Conference on Signals, Systems, and Computers, pp. 772-776, Nov. 2004.

[10] H. Tang, "Some Physical Layer Issues of Wide-Band Cognitive Radio Systems," in Proc. IEEE International Symp. Dynamic Spectrum Access Networks, DySPAN '05, pp. 151-159, Nov. 2005.

[11] S. Shankar, C. Cordeiro, and K. Challapali, "Spectrum Agile Radios: Utilization and Sensing Architectures," in Proc. IEEE International Symp. Dynamic Spectrum Access Networks, DySPAN '05, pp. 160- 169, Nov. 2005.

[12] W. Lee, I. F. Akyildiz, "Optimal Spectrum Sensing Framework for Cognitive Radio Networks," to appear in IEEE Transaction on Wireless Communications, 2008.

[13] A. Ghasemi, E.S. Sousa, "Collaborative spectrum sensing for opportunistic access in fading environments," in Proc. of First IEEE International Symposium on Dynamic Spectrum Access Networks, DySPAN '05, pp. 131-136, Nov. 2005.

[14] H. Urkowitz, "Energy detection of unknown deterministic signals," in Proc. of IEEE, vol. 55, pp.523-231, April 1967.

[15] K. Cai, V. Phan, and R. O'Connor, "Energy detector performance in a noise fluctuating channel," in Proc. of Military Communications Conference, MILCOM '89, pp.85-89, Oct.1989.

[16] J. Hillenbrand, T. A. Weiss and F. Jondral, Calculation of detection and false alarm probabilities in spectrum pooling systems, IEEE Communications Letters, vol. 9, no. 4, pp. 349C351, April 2005.

[17] S. Haykin, "Cognitive Radio: Brain-Empowered Wireless Communications," IEEE Journal on Selected Areas in Communications, vol. 23, no. 2, pp. 201C220, Feb. 2005.

[18] G. Ganesan, Y. Li, "Cooperative Spectrum Sensing in Cognitive Radio, Part I: Two User Networks," IEEE Transactions on Wireless Communications, vol.6, pp. 2204 - 2213, June 2007.

[19] G. Ganesan, Y. Li, "Cooperative Spectrum Sensing in Cognitive Radio, Part II: Multiuser Networks," IEEE Transactions on Wireless Communications, vol.6, pp. 2214 - 2222, June 2007.
[20] A. Rubinstein and M.Osborne, Bargaining and Markets, Academic Press, 1990.

[21] K. Sriram and W. Whitt, "Characterizing Superposition Arrival Processes in Packet Multiplexers for Voice and Data," IEEE Journal on Selected Areas in Communications, vol. SAC-4, pp. 833-846, Sep. 1986.

[22] IEEE 802.22 Working Group on Wireless Regional Area Networks, "IEEE P802.22/D0.1 Draft Standard for Wireless Regional Area Networks Part 22: Cognitive Wireless RAN Medium Access Control (MAC) and Physical Layer (PHY) specifications: Policies and procedures for operation in the TV Bands.", IEEE doc: 22-06-0067-00-0000_P80222_D0.1, May 2006.

[23] R. Chen, J. Park and K. Bian, "Robust Distributed Spectrum Sensing in Cognitive Radio Networks", in Proc. of INFOCOM '08, pp. 1876-1884, April 2008.

[24] T.S. Rappaport, Wireless communications: principles and practice, Prentice Hall, 1996.

[25] G. Chouinard, "IEEE P802.22 Wireless RANs: minutes of the 'channel model' sub-group teleconference," July 2005, available at: http://www.ieee802.org/22/.

[26] M. Gudmundson, "A correlation model for shadow fading in mobile radio," Electronic Letters, vol. 27, pp. 2146-2147, Nov. 1991. 